\title{
BMJ Open Treatment of hallux rigidus (HARD trial): study protocol of a prospective, randomised, controlled trial of arthrodesis versus watchful waiting in the treatment of a painful osteoarthritic first metatarsophalangeal joint
}

To cite: Miettinen M, Rämö L, Lähdeoja T, et al. Treatment of hallux rigidus (HARD trial): study protocol of a prospective, randomised, controlled trial of arthrodesis versus watchful waiting in the treatment of a painful osteoarthritic first metatarsophalangeal joint. BMJ Open 2021;11:e049298. doi:10.1136/ bmjopen-2021-049298

- Prepublication history and additional supplemental material for this paper are available online. To view these files, please visit the journal online (http://dx.doi.org/10.1136/ bmjopen-2021-049298).

Received 26 January 2021 Accepted 06 August 2021

Check for updates

(c) Author(s) (or their employer(s)) 2021. Re-use permitted under CC BY-NC. No commercial re-use. See rights and permissions. Published by BMJ.

For numbered affiliations see end of article.

Correspondence to Dr Mikko Miettinen; mikko.miettinen@hus.fi

\section{ABSTRACT}

Introduction Hallux rigidus is a common problem of pain and stiffness of the first metatarsophalangeal joint (MTPJ) caused mainly by degenerative osteoarthritis. Several operative techniques have been introduced for the treatment of this condition without high-quality evidence comparing surgical to non-surgical care. In this trial, the most common surgical procedure, arthrodesis, will be compared with watchful waiting in the management of hallux rigidus.

Methods and analysis Ninety patients (40 years or older) with symptomatic first MTPJ osteoarthritis will be randomised to arthrodesis or watchful waiting in a ratio of $1: 1$. The primary outcome will be pain during walking, assessed using the 0-10 Numerical Rating Scale (NRS) at 1 year after randomisation. The secondary outcomes will be pain at rest (NRS), physical function (ManchesterOxford Foot Questionnaire), patient satisfaction in terms of the patient-acceptable symptom state, health-related quality of life (EQ-5D-5L), activity level (The Foot and Ankle Ability Measure Sports subscale), use of analgesics or orthoses and the rate of complications. Our null hypothesis is that there will be no difference equal to or greater than the minimal important difference of the primary outcome measure between arthrodesis and watchful waiting. Our primary analysis follows an intention-to-treat principle.

Ethics and dissemination The study protocol has been approved by the Ethics Committee of Helsinki and Uusimaa Hospital District, Finland. Written informed consent will be obtained from all the participants. We will disseminate the findings of this study through peer-reviewed publications and conference presentations.

Protocol version 21 June 2021 V.2.0.

Trial registration number NCT04590313.

\section{INTRODUCTION}

Background and rationale

Hallux rigidus as a clinical diagnosis predates modern imaging methods-it refers to a stiff and painful first metatarsophalangeal joint
Strengths and limitations of this study

The study design is a prospective, randomised and controlled trial.

- To improve external validity, patients who decline randomisation will be followed as an observational cohort.

- We will use the gold standard technique performed by experienced consultant surgeons as the surgical intervention.

- We will use patient-reported and reliable, widely used outcome measures

- We consider variation of the waiting time for surgery a weakness as it causes difference in the recovery period between the surgery and the primary time point of 12 months.

(MTPJ). The most common condition associated with hallux rigidus is idiopathic osteoarthritis (OA) of the first MTPJ. Other less common predisposing conditions are inflammatory joint diseases and post-traumatic arthritis. ${ }^{1-3}$

The main symptoms and common findings are pain in the lift-off phase of gait, swelling or restricted extension of the MTPJ and a painful prominence on the dorsal side of the MTPJ. ${ }^{4}$ Pain usually begins at the extremes of the range of motion (ROM) but as the condition advances, also the midranges become painful and ROM becomes restricted. ${ }^{1}$ The diagnosis of hallux rigidus includes radiographic osteoarthritic findings in plain radiographs, including narrowing of the joint space and dorsal osteophyte formation. ${ }^{4}$

The prevalence of hallux rigidus has been reported to be $1.7 \%$ in a random population sample. ${ }^{5}$ Incidence of first MTPJ arthrodesis 
for treatment of hallux rigidus is ca. 20/100 000 personyears in Finland (Finnish National Hospital Discharge Registry).

The primary treatment of hallux rigidus is nonsurgical. At present, there is weak evidence supporting the role of orthoses and supportive shoes in the treatment of hallux rigidus. ${ }^{6}$ Currently, there is no comparative evidence supporting injection therapies, manipulation, physiotherapy interventions, extracorporeal shockwave therapy, iontophoresis and ultrasonography therapy. ${ }^{78}$ In a long-term cohort study of patients who declined surgery, pain levels remained constant in $92 \%$ of the patients in a 14-year follow-up. Nonetheless, $75 \%$ of these patients stated that they would make the same decision again. ${ }^{9}$

Several surgical treatment methods for hallux rigidus have been introduced, including arthrodesis, cheilectomy, osteotomy, implant arthroplasty, resection arthroplasty and interpositional arthroplasty. However, comparative evidence on the best surgical method is scarce. ${ }^{10}$ There are only two randomised controlled trials (RCTs) comparing surgical methods for hallux rigidus. In 2005 Gibson and Thomson compared arthrodesis and total joint replacement and in 2016 Baumhauer et al compared arthrodesis and synthetic cartilage implant. In both studies first MTPJ arthrodesis yielded better outcomes. ${ }^{11} 12$ Recommendations are based on expert opinion and panel consensus. ${ }^{13}$

Arthrodesis is widely accepted as the gold standard method for operative treatment of hallux rigidus. ${ }^{14-17}$ The spurs and cartilage of an affected joint are removed, and osteosynthesis is performed. ${ }^{18}$ However, there is no level I or II evidence on the best fixation method. The complication rate for arthrodesis has been presented to be less than $5 \% .{ }^{18}$ Most patients experience pain relief after arthrodesis, but the procedure also has disadvantages, such as decreased range of motion, shoe problems, long recovery and metatarsalgia. ${ }^{14-16}$

Currently, there is no comparative evidence on the effectiveness of surgery versus non-surgical care in the treatment of hallux rigidus. In a world of evidence-based medicine a gold standard intervention for a common condition without comparative evidence for efficacy is unacceptable. Our study is the first one designed to show the possible efficacy of surgery in treatment of hallux rigidus. Evidence on the efficacy of arthrodesis is required for any further studies (eg, cost-effectiveness) in this field and continuation of the treatment in clinical practice.

\section{Objectives and study hypothesis}

We will compare first MTPJ arthrodesis to watchful waiting in patients with at least a 1-year history of hallux rigidus due to idiopathic OA. Our null hypothesis is that there will be no difference equal to or greater than the minimal important difference of the primary outcome measure between arthrodesis and watchful waiting in treatment of hallux rigidus at 1 year.

\section{Trial design}

The trial will be a single-centre, parallel, two-armed 1:1, randomised open label, controlled superiority trial.

\section{METHODS}

\section{Study setting}

The study is based on a prospective cohort design. The randomised trial will be conducted at Helsinki University Hospital with a catchment area of 1.1 million people. The hospital is the largest orthopaedic foot and ankle centre in Finland, with over 500 first MTPJ arthrodesis performed annually. We will recruit patients at the consultant referral outpatient clinic of the foot and ankle unit.

\section{Patient and public involvement}

Patients were not involved in planning of research questions, outcome measures or design of the study.

\section{Eligibility criteria}

A member of the study group will assess the patients at the outpatient clinic (figure 1). We do not use classification systems when assessing eligibility, as they do not guide treatment and can be problematic in terms of reliability and validity. ${ }^{19}$ Eligible patients (box 1) will be introduced to the study and those willing to participate are asked to sign an informed consent form (see online supplemental file 1 for consent form).

Patients with bilateral hallux rigidus will be included in the trial with the more symptomatic foot. The operation of the other foot will be offered no earlier than 12 months after randomisation. When reporting the outcomes, the patients will be requested to consider the symptoms of the foot included in the trial.

\section{Interventions}

\section{Surgery}

The surgeries will be performed by experienced foot and ankle surgeons with experience of over 100 first MTPJ arthrodesis. Surgeries will be performed at 3-12 weeks after randomisation. Patients will receive an antibiotic prophylaxis (cefuroxime $1.5 \mathrm{~g}$ intravenous, or if contraindicated, clindamycin $600 \mathrm{mg}$ intravenous) before the operation. We will use supine patient positioning and a tourniquet pressure of $250 \mathrm{~mm} \mathrm{Hg}$. A medial approach will be used to remove joint surfaces manually or with a dome-shaped reamer. We will aim to achieve a $5^{\circ}-15^{\circ}$ valgus angulation and a neutral dorsal cortex alignment in the sagittal plane. ${ }^{2}$ In case of an unexpected large bone cyst, a bone autograft from the ipsilateral calcaneus will be used. We will use a compression screw and a dorsal locking plate for the fixation of the arthrodesis. This combination has shown superior results in terms of stability. ${ }^{21}{ }^{22}$ The joint capsule and skin will be closed with sutures. Patients will keep the postoperative dressing unopened for 2 days. Sutures will be removed after 14 days. We will instruct the patient 


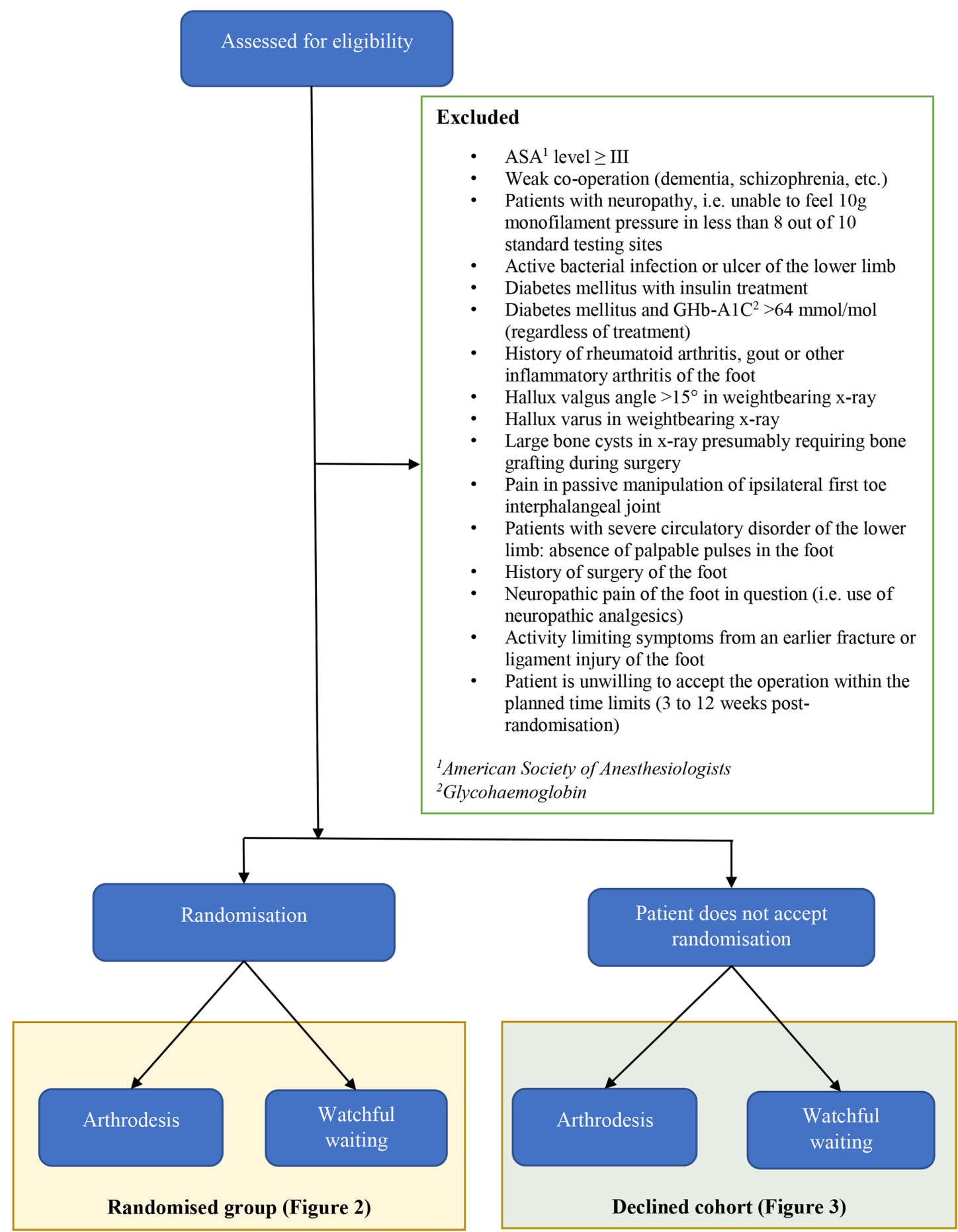

Figure 1 Flow chart of the enrolment and allocation in the HARD trial.

to wear a forefoot off-loading postoperative shoe for 6 weeks and allow immediate full weight-bearing with the shoe. $^{23}$

\section{Watchful waiting}

The patients randomised to the watchful waiting group will receive written and verbal information about hallux rigidus (online supplemental file 2), encouragement to keep an active lifestyle, and advice on the use of pain medication.

\section{Outcomes}

The outcome set of the HARD trial consists primarily of subjective patient-reported outcome measures (PROMs). We will record the outcomes (box 2) at baseline, 6 months and at 1,2 and 5 years.

\section{Baseline data}

The baseline data will be collected after informed consent but before randomisation. Baseline assessment will include information about sex, age, duration of 


\section{Box 1 Inclusion and exclusion criteria in the HARD trial}

\section{Inclusion criteria}

- Age 40 years or over.

- Diagnosis of hallux rigidus:

Persistent pain on movement of the first MTPJ*.

Osteoarthritic first MTPJ in plain X-rays (ie, narrowed joint space and osteophytes).

- Duration of symptoms $\geq 1$ year.

- Pain-NRS† during walking 4 or more on a scale $0-10$ (higher is worse).

- No substantial pain in other joints of the foot on non-clinical examination.

- Willingness to accept both treatment options.

- Ability to understand trial information and answer outcome assessments in Finnish.

- Signed informed consent.

Exclusion criteria

- ASA physical status classification level III or higher.

- Patients with weak cooperation (dementia, schizophrenia, etc).

- Patients with neuropathy, that is, unable to feel $10 \mathrm{~g}$ monofilament pressure in less than 8 out of 10 standard testing sites.

- Active bacterial infection or ulcer of the lower limb.

- Diabetes mellitus with insulin treatment.

- Diabetes mellitus and glycohaemoglobin (GHb-A1C)>64 mmol/mol (regardless of treatment).

- History of rheumatoid arthritis, gout or other inflammatory arthritis of the foot.

- Hallux valgus angle $>15^{\circ}$ in weight-bearing $X$-ray.

- Hallux varus in weight-bearing $X$-ray.

- Large bone cysts in $\mathrm{X}$-ray, presumably requiring bone grafting during surgery.

- Pain in passive manipulation of ipsilateral first toe interphalangeal joint.

- Patients with severe circulatory disorder of the lower limb: absence of palpable pulses in the foot (both dorsalis pedis artery and tibialis posterior artery).

- History of surgery of the foot in question.

- Neuropathic pain of the foot in question (ie, use of neuropathic analgesics).

- Activity limiting symptoms from an earlier fracture or ligament injury of the foot.

- Patient is unwilling to accept the operation within the planned time limits (3-12 weeks post-randomisation).

${ }^{*}$ Metatarsophalangeal joint.

†Numerical Rating Scale.

$\ddagger$ American Society of Anesthesiologists.

symptoms, history of smoking, education, occupation, comorbidities, use of analgesics and orthoses, Numerical Rating Scales (NRSs) for pain during walking, at rest during the day and at night, global disease rating, Pain Catastrophising Scale (PCS), the Manchester-Oxford Foot and Ankle Questionnaire (MOXFQ), ${ }^{24}$ the Foot and Ankle Ability Measure (FAAM) Sports subscale and the EQ-5D-5L health-related quality of life (HRQoL) questionnaire. ${ }^{25}$

All patients are screened with a $10 \mathrm{~g}$ monofilament for sensory neuropathy, and peripheral pulses are palpated. Hallux valgus, interphalangeal and intermetatarsal angles

\section{Box 2 Outcome measures in the HARD trial}

Primary outcome measure

1. Pain-Numerical Rating Scale (NRS) during walking at 1 year.*

Secondary outcome measures

1. Pain-NRS at rest.

2. Percentage of patients with acceptable symptom state patientacceptable symptom state.*

3. Complications.*

4. Manchester-Oxford Foot and Ankle Questionnaire Score.*

5. EQ-5D-5L Score.*

6. Foot and Ankle Ability Measure Sports Subscale.*

7. Use of analgesics and orthoses.

*See text for definition

are measured from X-rays. We will use a goniometer for measuring the range of motion of the first MTPJ. After the initial randomisation visit, all patients are further evaluated by weight-bearing CT (WBCT).

\section{Primary and secondary outcome measures}

Based on clinical experience, the major initial report among patients is pain during physical activity. ${ }^{26}$ The primary outcome measure of this study is pain during walking, assessed on the 0-10 NRS.

The primary time point is at 1 year after randomisation. Recent studies on first MTPJ surgery have shown only a minimal change in pain and PROMs after 6 months post-surgery. ${ }^{12} 27$ All outcome measures will be collected by paper forms at pre-specified time points described in table 1 .

\section{NRS for pain}

Pain at rest and during walking will be assessed on the 0-10 NRS, with 0 ('no pain') on the left and 10 ('worst possible pain') on the right. We consider 1.7 points on the NRS as minimal important difference (MID). ${ }^{28}$ The NRS is a reliable, valid and simple tool for assessing lower limb joint pain, with excellent correlation to the Visual Analogue Scale (VAS) ${ }^{29-31}$

Prospective global disability rating, patient acceptable symptom state, patient satisfaction

Patients will be asked to score their global disability rating on a 7 -item Likert scale. The question 'How much has your toe affected your normal daily activities, which require moving about, during the past week?' (Answer options: $1=$ not at all, $2=$ very slightly, a minor nuisance which did not affect my activities, $3=$ slightly, but enough that it mattered to me, $4=$ moderately, $5=$ moving around was very difficult, $6=$ moving around was extremely difficult, $7=$ could not move on my feet at all).

The proportion of patients reaching the Patient Acceptable Symptom State (PASS) will be determined by the question: 'If you think about your pain level and daily activities this week, would it be acceptable that your big toe would be like this for the rest of your life?. ${ }^{32} 33$ The 
Table 1 Assessments and interventions in the trial

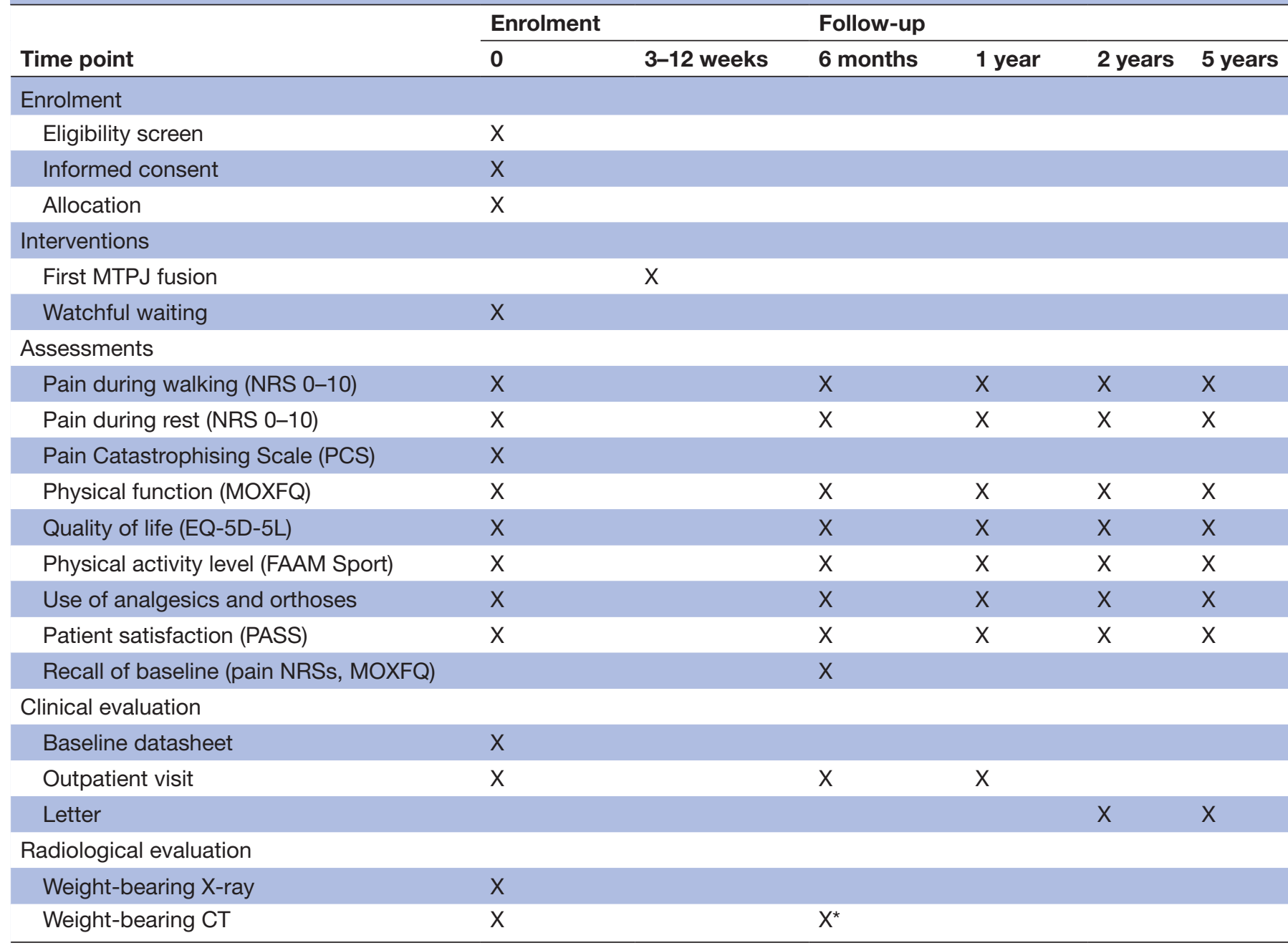

*Only the patients randomised to the surgery group.

FAAM, Foot and Ankle Ability Measure; MOXFQ, Manchester-Oxford Foot and Ankle Questionnaire; MTPJ, metatarsophalangeal joint; NRS, Numerical Rating Scale; PASS, patient acceptable symptom state.

answer options are 'Yes' or 'No'. The patients responding 'Yes' are considered to have reached PASS.

Patient satisfaction will be elicited by the question: 'How satisfied have you been with your big toe considering your daily activities and pain this week?' Options vary in 7-item Likert scale from 'very satisfied' (7) to 'very unsatisfied' (1).

\section{MOXFQ Score}

The MOXFQ is a PROM that was developed to evaluate the outcomes after treatment of hallux valgus. However, it has been shown to be generally valid for foot and ankle surgery. ${ }^{34-36}$ The instrument contains 16 items, and the response is given on a 5 -item Likert-scale, from 1 (worst) to 5 (best). The MOXFQ can be divided into three domains: walking/standing ( 7 items), pain (5 items) and social interaction ( 4 items). ${ }^{35}$ The scores of the subscales are scaled from 0 to 100 , where 0 represents low symptoms and 100 the most severe symptoms. ${ }^{37}$ In addition, it can be presented as a summary score. ${ }^{35}$ The minimally important difference has been estimated at 25 points for the pain and social interactions subscales, and 14 for the walking/standing subscale, using anchorbased methods. ${ }^{34}$ The results of the HARD trial will be given using the individual subscale scores.

\section{EQ-5D-5L Score}

The EuroQol instrument (EQ-5D-5L) is a HRQoL instrument divided into two parts: the EQ-5D descriptive system, and the EQ VAS. ${ }^{38} 39$ The EQ-5D descriptive system consist of five dimensions: mobility, self-care, usual activities, pain/discomfort and anxiety/depression. Each dimension has five response categories: no problems, some problems, moderate problems, severe problems and 'unable to'. The EQVAS includes an item about overall health stage on a 0-100 VAS.

\section{FAAM Sports Subscale Questionnaire}

The FAAM Sports subscale is a reliable, valid and responsive 8-item measure of self-reported physical 
function. ${ }^{40}$ It is possible that the patients adjust their activity levels to more sedentary to better cope with the foot pain, and this might be a confounding factor. We use the FAAM Sports subscale at all time points to assess patients' sport activity level to help assessing whether the groups remain comparable in this respect, and possibly to adjust analyses.

\section{Use of analgesics and orthoses}

Patients with painful conditions are prone to use pain medication and orthoses to alleviate their pain. These measures could possibly be a significant confounding factor in study with pain as a primary outcome. To reveal and to assess this possible confounding effect of an altering activity level and painkillers we will survey the use of analgesics and orthoses in all time points. The use of analgesics will be classified in four categories: (1) no use of analgesics or less frequent than weekly, (2) weekly use of analgesics, (3) daily use of nonsteroidal anti-inflammatory drugs (NSAIDs) or paracetamol and (4) daily use of opioids.

The use of orthoses will be collected as dichotomous variable (yes/no).

\section{PCS}

Patients with a tendency to catastrophise are reported to have more negative pain-related thoughts, greater emotional distress and greater pain intensity than noncatastrophisers. ${ }^{41}$ When answering pain-related questionnaires (such as the NRS, MOXFQ and EQ-5D-5L), catastrophisers tend to report more pain than noncatastrophisers. The PCS is a validated and reliable tool for assessing catastrophising. ${ }^{41} 42$

\section{Recall items}

We will estimate a recall error in transition items and explore response shift phenomenon. ${ }^{434}$ The patients will answer the pain questions and MOXFQ at the 6-month follow-up, recalling their situation when they agreed to participate in the trial.

\section{Safety considerations}

Adverse events in this study will be categorised as serious adverse events (SAEs) and minor adverse events (MAEs).

SAEs include but are not limited to death, cardiovascular events, bleeding requiring surgical intervention, symptomatic and ultrasound-verified deep venous thrombosis, CT-verified pulmonary embolism, deep infection of the operation site requiring reoperation and septic infection.

MAEs will include but are not limited to malunion (hallux valgus (HV) angle less than $0^{\circ}$ or more than $20^{\circ}$, sagittal dorsal cortex angle less than $-15^{\circ}$ or more than $+15^{\circ}$ ), non-union in CT at 6-month follow-up, implant failure and superficial infection with wound dehiscence of the operation site.

Information about adverse effects, concomitant care or interventions outside the study protocol, will be collected from medical records and during follow-up visits. All adverse events will be treated in the study hospital, by or under the supervision of an experienced orthopaedic foot and ankle surgeon.

The patients in the surgery groups will have an outpatient visit at 6 weeks after the operation. At this follow-up, the surgical wound and radiographs of the operated foot will be assessed. The bony union will be verified using the WBCT and the arthrodesis will be deemed non-united if no radiological consolidation is seen at 6 months. After this, symptomatic patients will be offered a reoperation.

\section{Possibility to crossover}

The patients in the watchful waiting group will have an opportunity to undergo arthrodesis if they do not get enough relief for their symptoms in 1 year. In these cases, the patients are operated with the same principles as the patients in the arthrodesis group.

\section{Participant timeline}

The timeline of enrolment, interventions, assessments and visits are shown in table 1 . A flow chart of the trial is presented in figure 2.

\section{Sample size}

The sample size calculation was performed using G*Power V.3.1 and was based on the primary outcome (pain-NRS during walking) ${ }^{45}$ We used $\alpha$-level of 0.05 and $\beta$-level of 0.15 and MID of 1.7 points (SD 2.5). ${ }^{28}$ The power calculation yielded the sample size of 40 per group with $85 \%$ power to show a difference equal to or larger than the MID between the treatments with a twosided type I error rate of $5 \%$. With the assumption of $11 \%$ lost to follow-up, we decided to include 45 participants per group.

\section{Allocation}

\section{Sequence generation and concealment}

The allocation sequence will be generated by a statistician with no clinical involvement in the execution of the trial. No stratification will be used. The research nurse, with no clinical involvement in the trial, will prepare identical, sequentially numbered and sealed envelopes according to the allocation sequence. The envelopes will be kept in a secure location at the study site. Randomisation will be performed in blocks of variable size, block structure known only by the statistician.

\section{Implementation of randomisation}

After receiving the informed consent, a member of the study group will open the next sequentially numbered envelope containing the treatment allocation.

\section{Declined cohort}

To increase the generalisability of our results and the external validity of the study, we will introduce a follow-up cohort of eligible patients declining randomisation (declined cohort). ${ }^{46}$ These patients will receive information about both treatment methods and can choose the treatment. The timeline for assessments and 


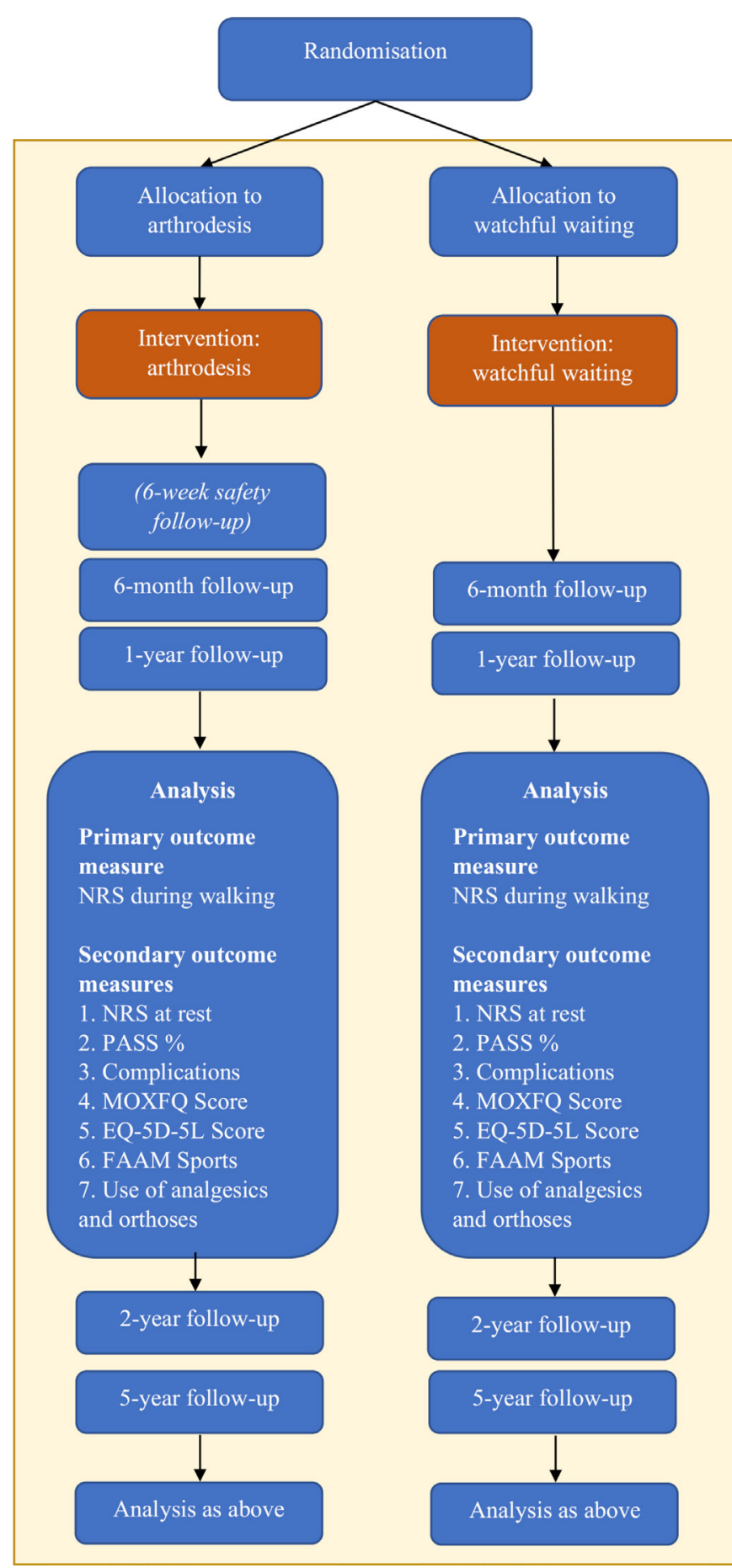

Figure 2 Flow chart of the interventions and follow-ups in the randomised cohort. FAAM, Foot and Ankle Ability Measure; MOXFQ, Manchester-Oxford Foot and Ankle Questionnaire; NRS, Numerical Rating Scale; PASS, patient acceptable symptom state; PCS, Pain Catastrophising Scale.

procedures of the declined cohort is shown in table 2. Each assessment will be performed with the same principles as in the randomised group, at the respective time points (figure 3). Analysis of the outcome measures will be done separately from the randomised cohort, and the results will be compared with the results of the RCT.
Data collection and management

Data will be collected using paper forms. On receiving the questionnaire forms, the researcher will make a visual check of the responses and will query missing data when possible. The paper forms will be securely stored at the study site.

We will use double data entry to reduce typing errors. Two persons not involved in the treatment of patients will enter the data to a database located in a secure network drive and protected with access codes known only by them. The two databases will be compared for consistency. Missing, implausible or inconsistent data in the electronic database will be checked from the original paper forms or the patient will be contacted. Final interpretation of the data will form the master database, which will be the source for the final data analyses.

\section{Statistical methods}

All primary and secondary analyses will be conducted according to the intention-to-treat principle. The results will be reported according to Consolidated Standards of Reporting Trials statement. ${ }^{47}$

The primary comparison (NRS-pain during walking) between the study groups will be performed using a mixed-model repeated-measures analysis of variance allowing missing data. Study group and time of assessment will be included as fixed factors and patients as random factors. Use of pain medication and orthoses, and FAAM Sports subscale will be used as covariates in the model to adjust the groups in terms of these possible confounding factors. The model includes interactions between study group and time of assessment. The model will be used to quantify the treatment effect as the absolute difference between the groups in pain-NRS during walking (mean and 95\% CI) and $p$ value at 12 months post-randomisation. We will consider two-sided $p$ value of 0.05 to indicate statistical significance.

Secondary outcomes will be compared using a similar model where applicable (pain-NRS at rest, MOXFQ, EQ-5D-5L, FAAM Sports). For categorical response variables, effects will be analysed using the generalised estimating equations model with the unstructured correlation structure. The secondary analyses will be considered only to be supportive, explanatory or hypothesis-generating (or both), which is why multiplicity is not considered a problem. Adverse events will be reported descriptively.

We plan to perform two sensitivity analyses: (1) perprotocol analysis with the crossover group, where patients are analysed as randomised only when they have been able to follow the pre-planned treatment protocol, and patients who have crossed over to the other treatment method will be analysed as a separate group and (2) as-treated analysis where the patients are analysed according to their current treatment method at each follow-up time point. In the as-treated analysis, the number of patients treated with surgery will increase in subsequent follow-up time points as some of the patients allocated to watchful waiting will receive operation during follow-up. 
Table 2 Assessments of the declined cohort

\begin{tabular}{|c|c|c|c|c|c|c|}
\hline \multirow[b]{2}{*}{ Time point } & \multirow{2}{*}{$\begin{array}{l}\text { Enrolment } \\
0\end{array}$} & \multirow{2}{*}{$\begin{array}{l}\text { Surgery } \\
3-12 \text { weeks }\end{array}$} & \multicolumn{4}{|l|}{ Follow-up } \\
\hline & & & 6 months & 1 year & 2 years & 5 years \\
\hline \multicolumn{7}{|l|}{ Enrolment } \\
\hline Informed consent & $\mathrm{X}$ & & & & & \\
\hline Allocation & $X$ & & & & & \\
\hline First MTPJ fusion & & $\mathrm{X}$ & & & & \\
\hline Watchful waiting & $x$ & & & & & \\
\hline \multicolumn{7}{|l|}{ Assessments } \\
\hline Pain during walking (NRS 0-10) & $x$ & & $x$ & $x$ & $x$ & $x$ \\
\hline Pain during rest (NRS 0-10) & $\mathrm{x}$ & & $\mathrm{x}$ & $\mathrm{X}$ & $x$ & $\mathrm{x}$ \\
\hline Physical activity level (FAAM Sport) & $\mathrm{x}$ & & $\mathrm{x}$ & $\mathrm{X}$ & $x$ & $\mathrm{x}$ \\
\hline Use of analgesics and orthoses & $x$ & & $x$ & $x$ & $x$ & $x$ \\
\hline Patient satisfaction (PASS) & $x$ & & $\mathrm{x}$ & $\mathrm{x}$ & $\mathrm{x}$ & $x$ \\
\hline Recall of baseline (pain NRSs, MOXFQ) & & & $x$ & & & \\
\hline \multicolumn{7}{|l|}{ Clinical evaluation } \\
\hline Baseline datasheet & $x$ & & & & & \\
\hline Outpatient visit & $\mathrm{x}$ & & $\mathrm{x}$ & & & \\
\hline Letter & & & & $x$ & $x$ & $x$ \\
\hline
\end{tabular}

*Only the patients in the surgery group.

FAAM, Foot and Ankle Ability Measure; MOXFQ, Manchester Oxford Foot and Ankle Questionnaire; MTPJ, metatarsophalangeal joint; NRS, Numerical Rating Scale; PASS, patient acceptable symptom state.

\section{Blinded data interpretation}

To avoid biased interpretation of the trial data, blinded data interpretation will be used in reporting the results of this trial. ${ }^{48}$ Before accessing the primary outcome data, the writing committee will record a 'Background assumptions' document containing our definition of MID of the outcome measures, and a brief summary of the key statistical analysis used in the evaluation of the outcome data. The document will be signed by the members of the writing committee and published as an appendix to the primary publication. After this, the writing committee will make two interpretations of the trial results based on a blinded review of the primary outcome data (treatment A compared with treatment B), with the assumption that $\mathrm{A}$ is the arthrodesis group and another assuming that $\mathrm{A}$ is the watchful waiting group. The writing committee will make the decisions regarding the key analyses and presentation format for the primary publication before data analysis. Before revealing the randomisation, the minutes of this meeting will be recorded and signed by all members of the writing committee. This document will be published as an appendix to the primary publication.

\section{Monitoring}

Data monitoring

We will conduct the study without a data monitoring committee. Both treatment methods are widely used in daily practice and have been proven to provide acceptable results. ${ }^{9} 18$ We will not conduct interim analyses.

Harms

All harms and complications of the treatment will be classified as MAEs or SAEs and reported in the publication of this trial.

\section{ETHICS AND DISSEMINATION}

Research ethics approval

This trial will be conducted according to the Helsinki Declaration. ${ }^{49}$ The protocol has been approved by the 


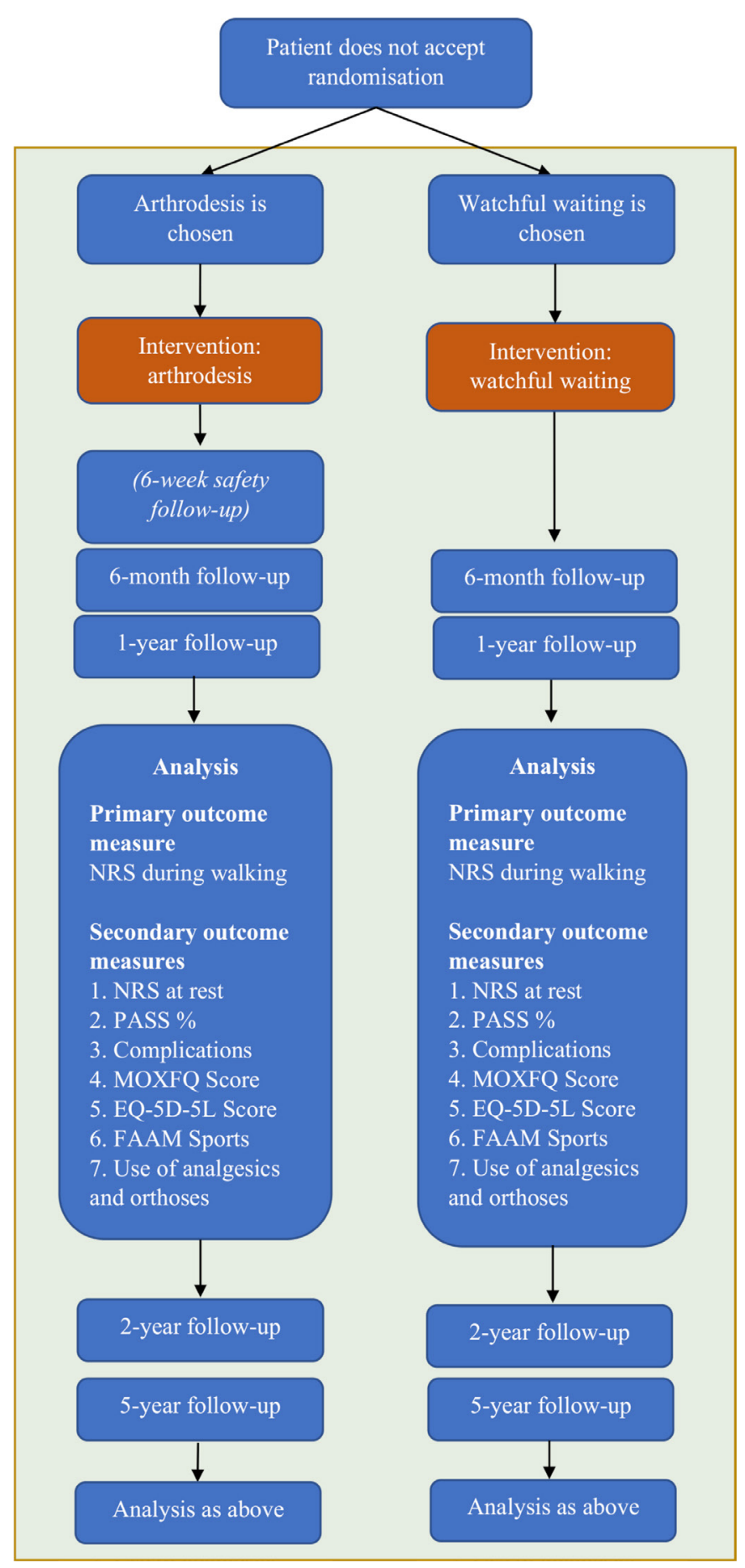

Figure 3 Flow chart of the interventions and follow-ups in the declined cohort. FAAM, Foot and Ankle Ability Measure; MOXFQ, Manchester-Oxford Foot and Ankle Questionnaire; NRS, Numerical Rating Scale; PASS, patient acceptable symptom state; PCS, Pain Catastrophising Scale.

institutional review board of the Helsinki and Uusimaa Hospital District (HUS/234/2020) and the trial has been duly registered at ClinicalTrials.gov.

\section{Protocol amendments}

All modifications of the study protocol will be updated in the trial registry.

\section{Consent}

The informed consent will be obtained by the recruiting members of the study group. The consent form (online supplemental file 1) is based on the General Data Protection Regulation Act of European Union (GDPR)compatible standardised form supplied by the Helsinki University Hospital. Consent will also be obtained from the participants of the declined cohort (online supplemental file 3 ).

\section{Confidentiality}

Trial data will be stored in a secure storage at the study centre for 15 years after completion of the study. All data will be handled according to the principles of the GDPR.

\section{Access to data}

The research nurses have exclusive access to the electronic trial data during data collection. The codes of the RCT arms will be known only to the research nurses until blinded data interpretation has taken place. Prior to publication of the final article, access to the primary data will be limited to statisticians, authors and reviewers. After publication the technical appendix, statistical code and data set will be available in the Dryad repository, DOI: https://doi.org/10.5061/dryad.vt4b8gtr.

\section{Ancillary and post-trial care}

Patients will be treated during and after the trial with best intention. If malpractice has taken place, patients will not receive any compensation beyond those from the Finnish Patient Insurance Centre.

\section{Dissemination policy}

The findings of this study will be disseminated through peer-reviewed publications and conference presentations and sent to participating patients.

\section{DISCUSSION}

We have described a study protocol of an RCT assessing the efficacy of fusion versus watchful waiting in the treatment of painful first MTPJ OA. ${ }^{50}$

To date there is no RCT comparing surgery with conservative treatment among patients with this condition. We chose to perform fusion with a plate and a lag screw as the surgical method in this trial since it is the most stable method of fixation according to the biomechanical studies. $^{21} 22$

As there is no gold standard for surgical treatment, we decided to compare the efficacy of surgery with the natural course of the disease. The isolation of the true efficacy of surgery would have required inclusion of a sham surgery group. We argue that in our study this would not have been feasible as the fused joint is obvious to the patient and blinding would probably be unsuccessful. Also, there is mounting evidence that the placebo effect of surgery is not very large, nor it is very long-lasting, and sham-controlled trials are not necessary if (a) there is no difference between surgery and non-surgical treatment 
in an open label trial or (b) the treatment difference is large. ${ }^{51} 52$

We as surgeons should appreciate that the patients' perception of the results is paramount. Therefore, we chose to use a PROM as the primary outcome of this trial and have included PROMs as secondary outcomes as well. We reserved the surgeon-based outcomes for adverse events only.

We consider the variation of the waiting time for surgery a possible weakness as it causes difference in the recovery period between surgery and the primary time point of 12 months. However, we have set the primary time point at 12 months to allow at least 9 months of recovery after the intervention, which we believe is sufficient for postoperative function and pain to reach a stable state. When planning the trial, we realised it was not possible to offer surgery in a fixed time point.

\section{Generalisability}

This study will be conducted in a foot and ankle unit of a university hospital. The surgeries will be performed by experienced consultant surgeons.

A common problem with RCTs is that only a small proportion of patients with a specific condition are included in the trial, potentially leading to poor external validity. ${ }^{46}$ Furthermore, among eligible patients, unwillingness to participate in randomisation may arise from a strong preference for one of the treatment modalities, causing participation bias. To assess the potential effect of participation bias we decided to follow eligible patients declining randomisation with the same protocol as the randomised cohort.

\section{Expectations}

We expect the primary outcome (the pain-NRS score during walking at 1 year after the randomisation) to be superior in the operative group.

In our expectations, the secondary outcome scores favour operative treatment as well, even though there will probably be more complications in the operative group in comparison to the watchful waiting group. We expect high PCS to correlate with worse outcome scores.

Recruitment will begin in 2021. At the expected recruiting pace, recruiting will end in 2024.

\section{Author affiliations}

${ }^{1}$ Department of Orthopaedics and Traumatology, University of Helsinki and Helsinki University Hospital, Helsinki, Uusimaa, Finland

${ }^{2}$ Finnish Centre for Evidence-Based Orthopaedics, FICEBO, Helsinki, Finland

${ }^{3}$ Department of Orthopaedics and Traumatology, Vaasa Central Hospital and University of Helsinki, Helsinki, Uusimaa, Finland

${ }^{4}$ Sports Hospital, Mehiläinen, Helsinki, Uusimaa, Finland

${ }^{5}$ Department of Orthopaedics and Traumatology, Central Finland Hospital Nova, Jyväskylä, Central Finland, Finland

${ }^{6}$ Department of Orthopaedics and Traumatology, University of Tampere and Tampere University Hospital, Tampere, Finland

Contributors MM, HS, LR, TL, TS, VP and JPR developed the trial, MM being the principal investigator. MM drafted the manuscript and all the members have actively contributed to the further writing of the manuscript. All authors have read and approved the final manuscript.
Funding The study is funded by The Research Foundation for Orthopaedics and Traumatology in Finland (grant number N/A) and University of Helsinki (grant number N/A). Investigators have not received any direct or indirect funding from the industry related to the topic of this study.

Competing interests None declared.

Patient consent for publication Not required.

Provenance and peer review Not commissioned; externally peer reviewed.

Supplemental material This content has been supplied by the author(s). It has not been vetted by BMJ Publishing Group Limited (BMJ) and may not have been peer-reviewed. Any opinions or recommendations discussed are solely those of the author(s) and are not endorsed by BMJ. BMJ disclaims all liability and responsibility arising from any reliance placed on the content. Where the content includes any translated material, BMJ does not warrant the accuracy and reliability of the translations (including but not limited to local regulations, clinical guidelines, terminology, drug names and drug dosages), and is not responsible for any error and/or omissions arising from translation and adaptation or otherwise.

Open access This is an open access article distributed in accordance with the Creative Commons Attribution Non Commercial (CC BY-NC 4.0) license, which permits others to distribute, remix, adapt, build upon this work non-commercially, and license their derivative works on different terms, provided the original work is properly cited, appropriate credit is given, any changes made indicated, and the use is non-commercial. See: http://creativecommons.org/licenses/by-nc/4.0/.

\section{ORCID iDs}

Mikko Miettinen http://orcid.org/0000-0003-0060-5360

Lasse Rämö http://orcid.org/0000-0003-4154-8462

Tuomas Lähdeoja http://orcid.org/0000-0002-9053-8888

Timo Sirola http://orcid.org/0000-0002-9287-3882

Henrik Sandelin http://orcid.org/0000-0003-2930-4182

Ville Ponkilainen http://orcid.org/0000-0002-5026-4560

Jussi P Repo http://orcid.org/0000-0002-4688-5698

\section{REFERENCES}

1 Lucas DE, Hunt KJ. Hallux rigidus relevant anatomy and pathophysiology. Foot and Ankle Clin 2015;20:381-9.

2 Ho B, Baumhauer J. Hallux rigidus. EFORT Open Rev 2017;2:13-20.

3 Brage ME, Ball ST. Surgical options for salvage of end-stage hallux rigidus. Foot Ankle Clin 2002;7:49-73.

4 Coughlin MJ, Shurnas PS. Hallux rigidus: demographics, etiology, and radiographic assessment. Foot Ankle Int 2003;24:731-43.

5 van Saase JL, van Romunde LK, Cats A, et al. Epidemiology of osteoarthritis: Zoetermeer survey. Comparison of radiological osteoarthritis in a Dutch population with that in 10 other populations. Ann Rheum Dis 1989;48:271-80.

6 Grady JF, Axe TM, Zager EJ, et al. A retrospective analysis of 772 patients with hallux limitus. J Am Podiatr Med Assoc 2002;92:102-8.

7 Kunnasegaran R, Thevendran G. Hallux rigidus: Nonoperative treatment and Orthotics. Foot Ankle Clin 2015;20:401-12.

8 Zammit G V, Menz HB, Munteanu SE. Interventions for treating osteoarthritis of the big toe joint. Cochrane Database Syst Rev 2010:CD007809. Sep 8.

9 Smith RW, Katchis SD, Ayson LC. Outcomes in hallux rigidus patients treated nonoperatively: a long-term follow-up study. Foot Ankle Int 2000;21:906-13.

10 Munteanu SE, Zammit GV, Menz HB, et al. Effectiveness of intraarticular hyaluronan (Synvisc, hylan G-F 20) for the treatment of first metatarsophalangeal joint osteoarthritis: a randomised placebocontrolled trial. Ann Rheum Dis 2011;70:1838-41.

11 Gibson JNA, Thomson CE. Arthrodesis or total replacement arthroplasty for hallux rigidus: a randomized controlled trial. Foot Ankle Int 2005;26:680-90.

12 Baumhauer JF, Singh D, Glazebrook M, et al. Prospective, randomized, Multi-centered clinical trial assessing safety and efficacy of a synthetic cartilage implant versus first Metatarsophalangeal arthrodesis in advanced hallux rigidus. Foot Ankle Int 2016;37:457-69.

13 McNeil DS, Baumhauer JF, Glazebrook MA. Evidence-Based analysis of the efficacy for operative treatment of hallux rigidus. Foot Ankle Int 2013;34:15-32.

14 Yee G, Lau J. Current concepts review: hallux rigidus. Foot Ankle Int 2008;29:637-46.

15 Fitzgerald JA, Wilkinson JM. Arthrodesis of the metatarsophalangeal joint of the great toe. Clin Orthop Relat Res 1981:70-7. 
16 Goucher NR, Coughlin MJ. Hallux metatarsophalangeal joint arthrodesis using dome-shaped reamers and dorsal plate fixation: a prospective study. Foot Ankle Int 2006;27:869-76.

17 Peace RA, Hamilton GA. End-Stage hallux rigidus: cheilectomy, implant, or arthrodesis? Clin Podiatr Med Surg 2012;29:341-53.

18 Coughlin MJ, Shurnas PS. Hallux rigidus. J Bone Joint Surg Am 2004:86-A Suppl 1:119-30.

19 Nixon DC, Lorbeer KF, McCormick JJ, et al. Hallux rigidus grade does not correlate with foot and ankle ability measure score. Journal of the American Academy of Orthopaedic Surgeons 2017;25:648-53.

20 Baumhauer JF, Singh D, Glazebrook M, et al. Correlation of hallux rigidus grade with motion, vas pain, intraoperative cartilage loss, and treatment success for first MTP joint arthrodesis and synthetic cartilage implant. Foot Ankle Int 2017;38:1175-82.

21 Politi J, John H, Njus G, et al. First metatarsal-phalangeal joint arthrodesis: a biomechanical assessment of stability. Foot Ankle Int 2003;24:332-7.

22 Doty J, Coughlin M, Hirose C, et al. Hallux Metatarsophalangeal joint arthrodesis with a hybrid locking plate and a plantar neutralization screw. Foot Ankle Int 2013;34:1535-40.

23 Navarro-Cano E, Guevara-Noriega KA, Lucar-Lopez G, et al. A comparison of two designs of postoperative shoe for hallux valgus surgery: a biomechanical study in a cadaveric model. Foot Ankle Surg 2021;27:82-6.

24 Dawson J, Doll H, Coffey J, et al. Responsiveness and minimally important change for the Manchester-Oxford foot questionnaire (MOXFQ) compared with AOFAS and SF-36 assessments following surgery for hallux valgus. Osteoarthritis Cartilage 2007;15:918-31.

25 Huskisson EC. Measurement of pain. The Lancet 1974;304:1127-31.

26 Hogan MV, Mani SB, Chan JY, et al. Validation of the foot and ankle outcome score for hallux rigidus. HSS Jrnl 2016;12:44-50.

27 Nilsdotter A-K, Cöster ME, Bremander A, et al. Patient-reported outcome after hallux valgus surgery - a two year follow up. Foot Ankle Surg 2019;25:478-81.

28 Olsen MF, Bjerre E, Hansen MD, et al. Pain relief that matters to patients: systematic review of empirical studies assessing the minimum clinically important difference in acute pain. BMC Med 2017;15:35

29 Alghadir AH, Anwer S, lqbal A, et al. Test-Retest reliability, validity, and minimum detectable change of visual analog, numerical rating, and verbal rating scales for measurement of osteoarthritic knee pain. J Pain Res 2018;11:851-6.

30 Landorf KB, Radford JA, Hudson S. Minimal important difference (mid) of two commonly used outcome measures for foot problems. J Foot Ankle Res 2010;3:7.

31 Farrar JT, Young JP, LaMoreaux L, et al. Clinical importance of changes in chronic pain intensity measured on an 11-point numerica pain rating scale. Pain 2001;94:149-58

32 Tubach F, Ravaud P, Martin-Mola E, et al. Minimum clinically important improvement and patient acceptable symptom state in pain and function in rheumatoid arthritis, ankylosing spondylitis, chronic back pain, hand osteoarthritis, and hip and knee osteoarthritis: results from a prospective multina. Arthritis Care Res 2012;64:1699-707.
33 Tubach Fet al. Evaluation of clinically relevant states in patient reported outcomes in knee and hip osteoarthritis: the patient acceptable symptom state. Ann Rheum Dis 2005;64:34-7.

34 Dawson J, Boller I, Doll H, et al. The MOXFQ patient-reported questionnaire: assessment of data quality, reliability and validity in relation to foot and ankle surgery. Foot 2011;21:92-102.

35 Morley D, Jenkinson C, Doll H, et al. The Manchester-Oxford foot questionnaire (MOXFQ). Bone Joint Res 2013;2:66-9.

36 Ponkilainen VT, Miettinen M, Sandelin $\mathrm{H}$, et al. Structural validity of the Finnish Manchester-Oxford foot questionnaire (MOXFQ) using the Rasch model. Foot and Ankle Surgery 2021;27:93-100.

37 Gallagher EJ, Liebman M, Bijur PE. Prospective validation of clinically important changes in pain severity measured on a visual analog scale. Ann Emerg Med 2001;38:633-8.

38 Brooks R, De Charro F. EuroQol: the current state of play. Health Policy 1996;37:53-72.

39 EuroQol. EQ-5D user guides, 2020. Available: https://euroqol.org/ publications/user-guides/

40 Martin RL, Irrgang JJ, Burdett RG, et al. Evidence of validity for the foot and ankle ability measure (FAAM). Foot Ankle Int 2005;26:968-83.

41 Sullivan MJL, Bishop SR, Pivik J. The pain Catastrophizing scale: development and validation. Psychol Assess 1995;7:524-32.

42 Rosenstiel AK, Keefe FJ. The use of coping strategies in chronic low back pain patients: relationship to patient characteristics and current adjustment. Pain 1983;17:33-44.

43 Razmjou H, Schwartz CE, Yee A, et al. Traditional assessment of health outcome following total knee arthroplasty was confounded by response shift phenomenon. J Clin Epidemiol 2009;62:91-6.

44 Razmjou H, Schwartz CE, Holtby R. The impact of response shift on perceived disability two years following rotator cuff surgery. $J$ Bone Joint Surg Am 2010;92:2178-86.

45 Faul F, Erdfelder E, Buchner A, et al. Statistical power analyses using $\mathrm{G}^{*}$ Power 3.1: tests for correlation and regression analyses. Behav Res Methods 2009;41:1149-60.

46 Reeves D, Howells K, Sidaway M, et al. The cohort multiple randomized controlled trial design was found to be highly susceptible to low statistical power and internal validity biases. $J$ Clin Epidemiol 2018;95:111-9.

47 Schulz KF, Altman DG, Moher D, et al. CONSORT 2010 statement: updated guidelines for reporting parallel group randomised trials. BMJ 2010;340:c332.

48 Järvinen TLN, Sihvonen R, Bhandari M, et al. Blinded interpretation of study results can feasibly and effectively diminish interpretation bias. J Clin Epidemiol 2014;67:769-72.

49 World Medical Association. World Medical association Declaration of Helsinki: ethical principles for medical research involving human subjects. JAMA 2013;310:2191-4.

50 Singal AG, Higgins PDR, Waljee AK. A primer on effectiveness and efficacy trials. Clin Trans/ Gastroenterol 2014;5:e45.

51 Beard DJ, Campbell MK, Blazeby JM, et al. Considerations and methods for placebo controls in surgical trials (ASPIRE guidelines). The Lancet 2020;395:828-38.

52 Hróbjartsson A, Gøtzsche PC. Placebo interventions for all clinical conditions. Cochrane Database Syst Rev 2010;43:CD003974. 